\title{
STABILITY AND CONTROL SYNTHESIS FOR DISCRETE-TIME LINEAR SYSTEMS SUBJECT TO ACTUATOR SATURATION BY OUTPUT FEEDBACK
}

\author{
A. BENZAOUIA, F. MESQUINE, A. HMAMED, AND H. AOUFOUSSI \\ Received 16 August 2004; Revised 15 September 2005; Accepted 22 September 2005
}

This paper presents sufficient conditions of asymptotic stability for discrete-time linear systems subject to actuator saturations with an output feedback law. The derived stability results are given in terms of LMIs. A new proof is presented to obtain previous conditions of asymptotic stability. A numerical example is used to illustrate this technique by using a linear optimization problem subject to LMI constraints.

Copyright (C) 2006 A. Benzaouia et al. This is an open access article distributed under the Creative Commons Attribution License, which permits unrestricted use, distribution, and reproduction in any medium, provided the original work is properly cited.

\section{Introduction}

It is well known that the problem of actuator saturation is inherent to all dynamical systems. This main problem has been an active area of research for many years $[7,17,26]$. Two main approaches have been developed in the literature. The first, the so-called positive invariance approach (see $[4,5,8,25]$ and the references therein), is based on the design of stabilizing controllers that work inside a region of linear behavior and do not allow saturation to be reached. An extension of this approach to a class of hybrid systems can be found in $[2,3,9]$. For the second approach however, the actuator saturations are allowed and the goal to achieve is the asymptotic stability despite the actuator saturations (see [20-22] and the references therein). The main objective of these two approaches is to obtain a domain of initial states as large as possible such that asymptotic stability is achieved despite the control constraints $[1,2,16,22]$.

On the other hand, the problem of stabilizing linear systems by output feedback, despite its apparent simplicity, is still open. A number of numerical procedures have been proposed for solving the problem since the work of Kimura [23, 24]. A survey was given by [27], and recent progress has been made for the related problem of pole placement (see $[13,15]$ and the references therein). However, less works were proposed for linear systems with actuator saturations. In [18] a dynamic output feedback is considered while in $[6,11]$ the positive invariance approach is used.

Hindawi Publishing Corporation

Mathematical Problems in Engineering

Volume 2006, Article ID 43970, Pages 1-11

DOI 10.1155/MPE/2006/43970 
This paper studies the static output feedback problem for discrete-time linear systems subject to actuator saturations. This work can be seen as an extension of the results of $[10,22]$ where a state feedback is used. The use of a key lemma rewriting the saturation function under a convex combination is the cornerstone of this work and the works of $[14,20-22]$. A different proof of the main result of [22] is obtained by using this writing of the saturation. Hence, a new sufficient condition of asymptotic stability for these systems is obtained. Further, the synthesis of the stabilizing controller by static output feedback is then proposed under LMI form. Furthermore, a relaxation technique is used to obtain less conservative LMIs. The proposed technique is completely different from all the previous works cited before on the same subject. The obtained regions of invariance and contractivity are generally less conservative.

This paper is organized as follows. The problem formulation is dealt with in the second section. The third section is reserved to some preliminary results useful for the development below. The main results of this work which consist in designing saturating stabilizing static output feedback for linear discrete-time systems with actuator saturations are presented in the fourth section. Section 5 deals with two illustrative examples.

\section{Problem formulation}

Consider the linear system described by

$$
\begin{gathered}
x_{k+1}=A x_{k}+B \text { sat }\left(u_{k}\right), \\
y_{k}=C x_{k},
\end{gathered}
$$

where $x_{k} \in \mathbb{R}^{n}$ is the state, $u_{k} \in \mathbb{R}^{m}$ is the control with $m \leq n, y_{k} \in \mathbb{R}^{p}$ is the output vector. The notation sat $(\cdot)$ stands for the standard saturation function assumed here to be normalized, that is, $\left|\operatorname{sat}\left(u_{k}\right)\right|=\min \left\{1,\left|u_{k}\right|\right\}$.

Let the following standard assumptions for the problem hold true:

(H1) matrix $C$ has full rank;

(H2) $(\mathrm{A}, \mathrm{B})$ is stabilizable; $(\mathrm{C}, \mathrm{A})$ is detectable.

Consider the following static output feedback control law:

$$
u_{k}=K y_{k}
$$

The closed-loop system is then given by

$$
x_{k+1}=A x_{k}+B \text { sat }\left(K C x_{k}\right) \text {. }
$$

In this work, we are interested in the synthesis of stabilizing controllers for linear systems subject to actuator saturations by static output feedback.

\section{Preliminary results}

In this section, two results on which our work is based are recalled, that is, a condition of asymptotic stability of a linear system subject to actuator saturation [10,22], together with a useful lemma. 
A. Benzaouia et al. 3

Define the following subsets of $\mathbb{R}^{n}$ :

$$
\begin{aligned}
\mathcal{E}(P, \rho) & =\left\{x \in \mathbb{R}^{n} \mid x^{T} P x \leq \rho, \rho>0\right\}, \\
\mathscr{L}(F) & =\left\{x \in \mathbb{R}^{n}|| f_{j} x \mid \leq 1,1 \leq j \leq m\right\},
\end{aligned}
$$

with $P$ a positive definite matrix and $f_{j}$ the $j$ th row of the matrix $F \in \mathbb{R}^{m \times n} \cdot \varepsilon(P, \rho)$ is an ellipsoid set while $\mathscr{L}(F)$ is a polyhedral set for which the saturation does not occur.

Theorem 3.1 [22]. Given an ellipsoid $\varepsilon(P, \rho)$, if there exists a matrix $H \in \mathbb{R}^{m \times n}$ such that

$$
\left[A+B\left(D_{i} K C+D_{i}^{-} H\right)\right]^{T} P\left[A+B\left(D_{i} K C+D_{i}^{-} H\right)\right]-P<0, \quad \forall i \in\left[1,2^{m}\right],
$$

and $\varepsilon(P, \rho) \subset \mathscr{L}(H)$, then $\varepsilon(P, \rho)$ is a contractively invariant set for the closed-loop saturated system in (2.3).

Matrices $D_{i}$ are $m$-by- $m$ diagonal matrices with elements either 1 or 0 and $D_{i}^{-}=\rrbracket_{m}-$ $D_{i}$. There are $2^{m}$ possible combinations with 1 and 0 leading to $2^{m}$ different matrices $D_{i}$. Note that matrices $D_{i}$ and $D_{i}^{-}$are introduced by [22] to model the nonlinear saturation function as a linear convex combination by using the following lemma.

Lemma 3.2 [22]. For all $u \in \mathbb{R}^{m}$ and $v \in \mathbb{R}^{m}$ such that $\left|v_{j}\right|<1, j \in[1, m]$,

$$
\operatorname{sat}(u) \in \operatorname{co}\left\{D_{i} u+D_{i}^{-} v, i \in \mathscr{I}\right\}
$$

with $\mathscr{I}=[1, \eta], \eta=2^{m}$, and co standing for the convex hull. In this case, there exist $\delta_{1} \geq 0, \ldots, \delta_{\eta} \geq 0$ satisfying $\sum_{i=1}^{\eta} \delta_{i}=1$ such that

$$
\operatorname{sat}(u)=\sum_{i=1}^{\eta} \delta_{i}\left[D_{i} u+D_{i}^{-} v\right] .
$$

\section{Main result}

In this section, the design of stabilizing controllers for linear systems with actuator saturations is presented by using the results of both Theorem 3.1 and Lemma 3.2. A different proof of Theorem 3.1 is also proposed. Note that $*$ is used to write the transpose of the off-diagonal element of an LMI.

Theorem 4.1. For a given positive scalar $\rho$, if there exists a symmetric matrix $P$ and $a$ matrix $H$ such that

$$
\begin{gathered}
{\left[\begin{array}{cc}
P & {\left[A+B\left(D_{i} K C+D_{i}^{-} H\right)\right]^{T} P} \\
* & P
\end{array}\right]>0, \quad \forall i \in \mathscr{I}} \\
\mathcal{E}(P, \rho) \subset \mathscr{L}(H),
\end{gathered}
$$

then the closed-loop system (2.3) is asymptotically stable for all $x_{0} \in \varepsilon(P, \rho)$.

Proof. Assume that there exists a matrix $H$ and a symmetric matrix $P$ such that conditions (4.1) and (4.2) hold true. Using Lemma 3.2, there exist $\delta_{1}, \ldots, \delta_{\eta}$ such that one can rewrite 
4 Output feedback for saturated systems

the saturated output feedback control (2.2) as follows:

$$
\begin{gathered}
\operatorname{sat}\left(K C x_{k}\right)=\sum_{i=1}^{\eta} \delta_{i}(k)\left[D_{i} K C+D_{i}^{-} H\right] x_{k} ; \\
\delta_{i}(k) \geq 0, \quad \sum_{i=1}^{\eta} \delta_{i}(k)=1 .
\end{gathered}
$$

The closed-loop system can be rewritten as

$$
\begin{gathered}
x_{k+1}=\sum_{i=1}^{\eta} \delta_{i}(k) A c_{i} x_{k} ; \\
A c_{i}=A+B\left(D_{i} K C+D_{i}^{-} H\right), \quad i \in \mathscr{I} .
\end{gathered}
$$

Consider now the Lyapunov function candidate given by

$$
V\left(x_{k}\right)=x_{k}^{T} P x_{k}
$$

Its rate of increase on the trajectories of the system (4.4) is given by

$$
\Delta V\left(x_{k}\right)=x_{k+1}^{T} P x_{k+1}-x_{k}^{T} P x_{k}=x_{k}^{T}\left\{\left[\sum_{i=1}^{\eta} \delta_{i}(k) A c_{i}\right]^{T} P\left[\sum_{i=1}^{\eta} \delta_{i}(k) A c_{i}\right]-P\right\} x_{k} .
$$

Recall that condition (4.1) is satisfied and premultiply each inequality $(4.1)$ for $i=1, \ldots, \eta$ by $\delta_{i}(k)$. Summing up the obtained inequalities and bearing in mind that $\sum_{i=1}^{\eta} \delta_{i}(k)=1$, one gets

$$
\left[\begin{array}{cc}
P & {\left[\sum_{i=1}^{\eta} \delta_{i}(k) A c_{i}\right]^{T} P} \\
* & P
\end{array}\right]>0 .
$$

The use of Schur complement allows one to write condition (4.7) under the equivalent form,

$$
\left[\sum_{i=1}^{\eta} \delta_{i}(k) A c_{i}\right]^{T} P\left[\sum_{i=1}^{\eta} \delta_{i}(k) A c_{i}\right]-P<0, \quad \forall i \in \mathscr{I},
$$

which ensures that

$$
\Delta V\left(x_{k}\right)<-\gamma\left(\left\|x_{k}\right\|\right)
$$

where

$$
\gamma\left(\left\|x_{k}\right\|\right)=\min _{i} \lambda_{\min }\left(P-\left[\sum_{i=1}^{\eta} \delta_{i}(k) A c_{i}\right]^{T} P\left[\sum_{i=1}^{\eta} \delta_{i}(k) A c_{i}\right]\right)\left\|x_{k}\right\|^{2} .
$$


Taking into account condition (4.2) and noticing that $\varepsilon(P, \rho)$ is a contractively invariant set, one can guarantee that for all $x_{o} \in \varepsilon(P, \rho) \subset \mathscr{L}(H)$, the saturated system (2.3) is asymptotically stable.

Note that Theorem 4.1 proposes a different proof of [22, Theorem 3.1] for linear systems with state feedback by letting $C=\square$. This result of stability can be exploited for the synthesis of the controller by the following.

THeOREM 4.2. For a given scalar $\rho$, if there exists a symmetric matrix $X \in \mathbb{R}^{n \times n}$, matrices $V \in \mathbb{R}^{p \times p}, Y \in \mathbb{R}^{m \times p}$, and $Z \in \mathbb{R}^{m \times n}$ solutions of the LMIs

$$
\begin{gathered}
{\left[\begin{array}{cc}
X & \left(A X+B D_{i} Y C+B D_{i}^{-} Z\right)^{T} \\
* & X
\end{array}\right]>0, \quad \forall i \in \mathscr{I} ;} \\
{\left[\begin{array}{cr}
\frac{1}{\rho} & z_{j} \\
* & X
\end{array}\right]>0, \quad \forall j \in[1, m] ;} \\
V C-C X=0,
\end{gathered}
$$

then, the closed-loop system subject to saturations (2.3) is asymptotically stable at the origin for all $x_{0} \in \varepsilon(P, \rho)$, where $z_{j}$ is the $j$ th row of matrix $Z$ and

$$
\begin{gathered}
K=Y V^{-1}, \\
H=Z X^{-1} \\
P=X^{-1}
\end{gathered}
$$

Proof. Let conditions (4.11)-(4.13) hold true. According to (4.13), one can write $K C X=$ $K V C$. Since matrix $X$ is positive definite, and by virtue of assumption $(H 1)$, matrix $V$ solution of (4.13) is nonsingular. Further, by replacing $Y=K V$ and $Z=H X$ as given by (4.14)-(4.15), the LMI,

$$
\left[\begin{array}{cc}
X & \left(A X+B D_{i} K C X+B D_{i}^{-} H X\right)^{T} \\
* & X
\end{array}\right]>0
$$

the use of the Schur complement leads to follows.

$$
\left[A+B\left(D_{i} K C+D_{i}^{-} H\right)\right] X\left[A+B\left(D_{i} K C+D_{i}^{-} H\right)\right]^{T}-X<0 .
$$

Since $X=P^{-1}$, inequality (4.19) becomes

$$
\left[A+B\left(D_{i} K C+D_{i}^{-} H\right)\right] P^{-1}\left[A+B\left(D_{i} K C+D_{i}^{-} H\right)\right]^{T}-P^{-1}<0 .
$$

Postmultiplying and premultiplying the last inequalities by $P$ leads to

$$
P\left[A+B\left(D_{i} K C+D_{i}^{-} H\right)\right] P^{-1}\left[A+B\left(D_{i} K C+D_{i}^{-} H\right)\right]^{T} P-P<0 .
$$


The use of the Schur complement a second time leads to LMI (4.1). Using [19], the LMI (4.12) can also be transformed to the equivalent inclusion condition (4.2) with $X=P^{-1}$. Consequently, by virtue of Theorem 4.1, the closed-loop system subject to saturations (2.3) is asymptotically stable at the origin for all $x_{0} \in \varepsilon(P, \rho)$.

It is worth noting that the state feedback problem follows readily from Theorem 4.2 by letting $C=\rrbracket_{m}$. In this case, $V=X$. The resolution of these LMIs can be extended to the case where the scalar $\rho$ is also taken as a design variable.

Conditions of Theorem 4.2 may be conservative due to (4.13). In order to relax this conservatism, we associate this equation to a second matrix $S$ different from $X$ as suggested by [12] for unsaturated systems. This technique is presented by the following result.

THeOREM 4.3. For a given scalar $\rho$, if there exist symmetric matrix $X \in \mathbb{R}^{n \times n}$, matrices $V \in \mathbb{R}^{p \times p}, S \in \mathbb{R}^{n \times n}, Y \in \mathbb{R}^{m \times p}$, and $Z \in \mathbb{R}^{m \times n}$ solutions of the LMIs

$$
\begin{gathered}
{\left[\begin{array}{cc}
S+S^{T}-X & {\left[A S+B D_{i} Y C+B D_{i}^{-} Z\right]^{T}} \\
* & X
\end{array}\right]>0, \quad \forall i \in \mathscr{I},} \\
{\left[\begin{array}{cr}
\frac{1}{\rho} & z_{j} \\
* & X
\end{array}\right]>0, \quad \forall j \in[1, m],} \\
V C-C S=0,
\end{gathered}
$$

then the closed-loop system subject to saturations (2.3) is asymptotically stable at the origin for all $x_{0} \in \varepsilon(P, \rho)$, where $z_{j}$ is the $j$ th row of matrix $Z$ and

$$
\begin{gathered}
K=Y V^{-1}, \\
H=Z S^{-1} \\
P=X^{-1}
\end{gathered}
$$

Proof. The main idea of this proof is given by [12]. Using (4.23), (4.24), and (4.25), the LMI (4.21) can be rewritten equivalently as

$$
\left[\begin{array}{cc}
S+S^{T}-X & S^{T}\left(A+B D_{i} K C+B D_{i}^{-} H\right)^{T} \\
* & X
\end{array}\right]>0 .
$$

It is obvious that if (4.21) holds, then $S+S^{T}-X>0$; thus, matrix $S$ is nonsingular; hence matrix $V$ is also nonsingular. Since matrix $X$ is positive definite, we have

$$
(X-S)^{T} X^{-1}(X-S)>0 .
$$

This implies that

$$
S^{T} X^{-1} S \geq S+S^{T}-X .
$$


Inequalities (4.29) and (4.21) enable us to write

$$
\left[\begin{array}{cc}
S^{T} X^{-1} S & S^{T}\left(A+B D_{i} K C+B D_{i}^{-} H\right)^{T} \\
* & X
\end{array}\right]>0 .
$$

This LMI is equivalent to

$$
\left[\begin{array}{cc}
S^{T} & 0 \\
0 & X
\end{array}\right]\left[\begin{array}{cc}
X^{-1} & \left(A+B D_{i} K C+B D_{i}^{-} H\right)^{T} X^{-1} \\
* & X^{-1}
\end{array}\right]\left[\begin{array}{cc}
S & 0 \\
0 & X
\end{array}\right]>0 .
$$

Consequently, by letting $P=X^{-1}$, the LMI (4.21) implies the LMI (4.1). This last LMI, together with condition (4.22), presents the sufficient conditions of stability of Theorem 4.1. Hence, the closed-loop system subject to saturations (2.3) is asymptotically stable at the origin for all $x_{0} \in \varepsilon(P, \rho)$.

It is worth noting that in the case of output feedback we have more constraining equalities (4.13) that may generally lead to conservative regions of stability. Hence, as in the previous works on the problem of saturated systems, it is of great interest to enlarge the ellipsoid $\varepsilon(P, \rho)$ of initial conditions. Two optimization procedures to obtain the largest ellipsoid are proposed by rewriting the LMI (4.22) with $\mu=1 / \rho$ as an additional variable as follows:

$$
(P b .1):\left\{\begin{array}{l}
\inf _{(X, Y, V, Z)}(\mu) \\
\text { s.t. }(4.21),(4.22),(4.23) .
\end{array}\right.
$$

This optimization problem can help to enlarge the ellipsoids $\varepsilon(P, \rho)$ by maximizing the scalars $\rho$. Since the volume of the ellipsoid is proportional to the trace of matrix $X$, a second way to obtain larger sets of invariance and contractivity is to solve the following optimization problem:

$$
(P b .2):\left\{\begin{array}{l}
\sup _{(X, Y, V, Z)} \operatorname{Trace}(X) \\
\text { s.t. }(4.21),(4.22),(4.23)
\end{array}\right.
$$

According to [22], when this optimization problem is feasible, the volume of the obtained ellipsoids is maximum with respect to the data of the system. The resolution of these two optimization problems can be easily obtained by the use of Matlab.

\section{Examples}

In this section, two illustrative examples are presented to show the applicability of the results presented above.

Example 5.1. Consider the double integrator system modeled by the saturated discretetime linear system given by the following matrices:

$$
A=\left[\begin{array}{cc}
1 & 1 \\
0 & 1
\end{array}\right] ; \quad B=\left[\begin{array}{c}
0.5 \\
1
\end{array}\right] ; \quad C=\left[\begin{array}{ll}
1 & 1
\end{array}\right] .
$$




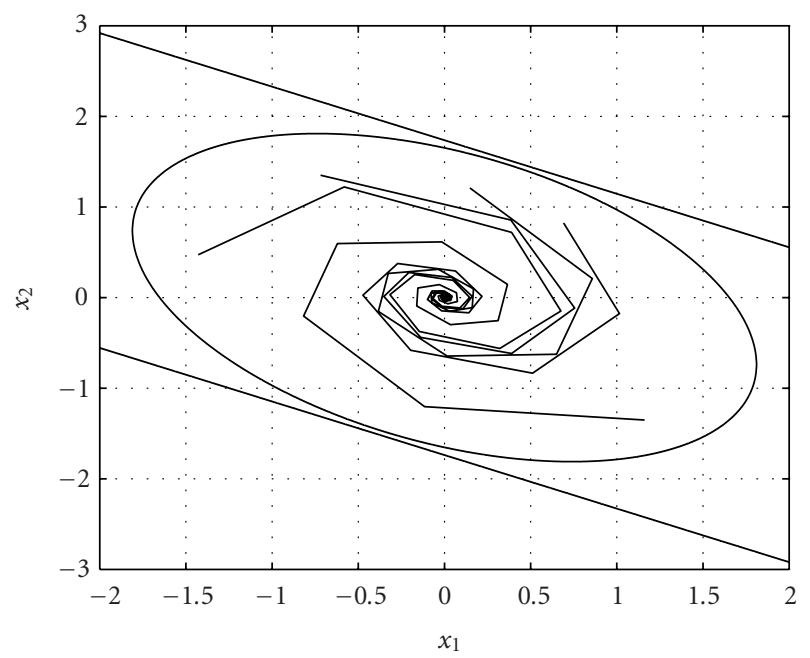

Figure 5.1. The ellipsoid set of invariance and contractivity for the saturated discrete-time linear system with output feedback with 5 trajectories.

For this example with $n=2$ and $m=p=1$, we have to solve the LMIs of Theorem 4.2 with 4 variables. Let the scalar $\rho$ be given equal to 1 . The use of the LMI Matlab Toolbox leads to the following:

$$
\begin{gathered}
P=\left[\begin{array}{ll}
0.3661 & 0.1494 \\
0.1494 & 0.3661
\end{array}\right] ; \\
H=\left[\begin{array}{ll}
-0.3402 & -0.5756
\end{array}\right] ; \quad K=-0.7826 \\
A c=A+B K C=\left[\begin{array}{cc}
0.6087 & 0.6087 \\
-0.7826 & 0.2174
\end{array}\right] ; \\
\sigma(A c)=\{0.4131+0.6619 i ; 0.4131-0.6619 i\} .
\end{gathered}
$$

Figure 5.1 presents the ellipsoid set of invariance and contractivity for the saturated discrete-time linear system with output feedback. Since the LMIs of Theorem 4.2 are feasible, the relaxation technique is not used for this example.

Example 5.2. Consider now the following example studied in [10]:

$$
A=\left[\begin{array}{ccc}
1 & 1 & 0.5 \\
0 & 1 & 1 \\
1 & 0 & 1
\end{array}\right] ; \quad B=\left[\begin{array}{c}
1.67 \\
0.5 \\
1
\end{array}\right] ; \quad C=\left[\begin{array}{ccc}
1 & 1 & 0 \\
0 & 0 & 1
\end{array}\right]
$$

For this example with $n=3, m=1$, and $p=2$, we have to solve the LMIs of Theorem 4.2. Let the scalar $\rho$ be given equal to 1 . The use of the LMI Matlab Toolbox leads to the 
A. Benzaouia et al. 9

following results:

$$
\begin{aligned}
& P=\left[\begin{array}{ccc}
0.5380 & -0.1923 & 0 \\
-0.1923 & 0.5380 & 0 \\
0 & 0 & 0.8555
\end{array}\right] \\
& H=\left[\begin{array}{lll}
-0.3656 & -0.2859 & -0.5397
\end{array}\right] \text {; } \\
& K=[-0.4122-0.4699] ; \quad V=\left[\begin{array}{cc}
2.8929 & 0 \\
0 & 1.1690
\end{array}\right] \text {; } \\
& A c=A+B K C=\left[\begin{array}{ccc}
0.3116 & 0.3116 & -0.2847 \\
-0.2061 & 0.7939 & 0.7650 \\
0.5878 & -0.4122 & 0.5301
\end{array}\right] \text {; } \\
& \sigma(A c)=\{0.4639+0.7131 i ; 0.4639-0.7131 i ; 0.7079\} \text {. }
\end{aligned}
$$

While we obtain with the LMIs of Theorem 4.3 the following solutions:

$$
\begin{aligned}
& P=\left[\begin{array}{lll}
0.1021 & 0.0901 & 0.1129 \\
0.0901 & 0.0929 & 0.1086 \\
0.1129 & 0.1086 & 0.1379
\end{array}\right] \\
& H=\left[\begin{array}{lll}
-0.4321 & -0.4024 & -0.5409
\end{array}\right] ; \quad K=[-0.5426-0.6904] ; \\
& S=\left[\begin{array}{ccc}
127.1701 & -20.3197 & -86.4222 \\
1.0455 & 148.5353 & -109.7623 \\
-103.2536 & -103.2536 & 162.8427
\end{array}\right] ; \quad V=\left[\begin{array}{cc}
128.2156 & -196.1846 \\
-103.2536 & 162.8427
\end{array}\right] \text {; } \\
& A c=A+B K C\left[\begin{array}{ccc}
0.0939 & 0.0939 & -0.6530 \\
-0.2713 & 0.7287 & 0.6548 \\
0.4574 & -0.5426 & 0.3096
\end{array}\right] \\
& \sigma(A c)=\{0.2770 ; 0.4276+0.7634 i ; 0.4276-0.7634 i\} \text {. }
\end{aligned}
$$

For this example, LMIs of both Theorems 4.2 and 4.3 were found feasible. Hence, the obtained results are slightly similar. Generally, the results of Theorem 4.3 are used when the LMIs of Theorem 4.2 are not feasible.

\section{Conclusion}

This work deals with asymptotic stability of linear systems with static output feedback subject to actuator saturations. Sufficient conditions of asymptotic stability are then established. Another proof of the results of [22] obtained with state feedback control is given in the case of output feedback control. This proof is based on the rewriting of the saturation function under a linear convex function. Further, all the results of this work are presented under LMI form. A relaxation technique, together with two optimization problems, is also given to improve the feasibility of the LMIs and the conservatism of the solutions. Two illustrative examples are studied to show the applicability of this approach. 


\section{Acknowledgment}

The authors would like to acknowledge the careful reviews of the referees whose comments have been very helpful in improving the overall quality of this paper.

\section{References}

[1] A. Benzaouia and A. Baddou, Piecewise linear constrained control for continuous-time systems, IEEE Transactions on Automatic Control 44 (1999), no. 7, 1477-1481.

[2] A. Benzaouia, A. Baddou, and S. Elfaiz, Piecewise linear constrained control for continuous-time systems: the maximal admissible domain, Proceedings of 15th IFAC World Congress, Barcelona, 2002.

[3] A. Benzaouia, E. L. Boukas, and N. Daraoui, Stability of continuous-time linear systems with Markovian jumping parameters and constrained control, Proceedings of 15th IFAC World Congress, Barcelona, 2002.

[4] A. Benzaouia and C. Burgat, Regulator problem for linear discrete-time systems with nonsymmetrical constrained control, International Journal of Control 48 (1988), no. 6, 2441-2451.

[5] A. Benzaouia and A. Hmamed, Regulator problem for linear continuous-time systems with nonsymmetrical constrained control, IEEE Transactions on Automatic Control 38 (1993), no. 10, $1556-1560$.

[6] A. Benzaouia and D. Mehdi, The output feedback saturated controller design for linear systems, Proceedings of the 10th Mediterranean Conferenceon Control and Automation, Lisbon, 2002.

[7] D. S. Bernstein and A. N. Michel, A chronological bibliography on saturating actuators, International Journal of Robust and Nonlinear Control 5 (1995), no. 5, 375-380.

[8] F. Blanchini, Set invariance in control, Automatica 35 (1999), no. 11, 1747-1767.

[9] E. K. Boukas and A. Benzaouia, Stability of discrete-time linear systems with Markovian jumping parameters and constrained control, IEEE Transactions on Automatic Control 47 (2002), no. 3, 516-521.

[10] Y.-Y. Cao and Z. Lin, Stability analysis of discrete-time systems with actuator saturation by a saturation-dependent Lyapunov function, Proceedings of 41st IEEE Conference on Decision and Control, vol. 4, Nevada, 2002, pp. 4140-4145.

[11] E. B. Castelan and S. Tarbouriech, On positive invariance and output feedback stabilization of input constrained linear systems, Proceedings of American Control Conference, vol. 3, Maryland, 1994, pp. 2740-2745.

[12] J. Daafouz, P. Riedinger, and C. Iung, Stability analysis and control synthesis for switched systems: a switched Lyapunov function approach, IEEE Transactions on Automatic Control 47 (2002), no. $11,1883-1887$.

[13] L. El Ghaoui, F. Oustry, and M. AitRami, A cone complementarity linearization algorithm for static output-feedback and related problems, IEEE Transactions on Automatic Control 42 (1997), no. $8,1171-1176$.

[14] H. Fang, Z. Lin, and T. Hu, Analysis of linear systems in the presence of actuator saturation and $\mathscr{L}_{2}$-disturbances, Automatica 40 (2004), no. 7, 1229-1238.

[15] G. Garcia, B. Pradin, and F. Zeng, Stabilization of discrete time linear systems by static output feedback, IEEE Transactions on Automatic Control 46 (2001), no. 12, 1954-1958.

[16] E. G. Gilbert and K. T. Tan, Linear systems with state and control constraints: the theory and application of maximal output admissible sets, IEEE Transactions on Automatic Control 36 (1991), no. $9,1008-1020$.

[17] C. Gökçek, P. T. Kabamba, and S. M. Meerkov, An LQR/LQG theory for systems with saturating actuators, IEEE Transactions on Automatic Control 46 (2001), no. 10, 1529-1542. 
[18] D. Henrion, S. Tarbouriech, and G. Garcia, Output feedback robust stabilization of uncertain linear systems with saturating controls: an LMI approach, IEEE Transactions on Automatic Control 44 (1999), no. 11, 2230-2237.

[19] H. Hindi and S. Boyd, Analysis of linear systems with saturating using convex optimization, Proceeding of the 37th IEEE Conference on Decision and Control, vol. 1, Florida, 1998, pp. 903-908.

[20] T. Hu and Z. Lin, The equivalence of several set invariance conditions under saturation, Procceding of the 41st IEEE Conference on Decision and Control, vol. 4, Nevada, 2002, pp. 4146-4147.

[21] T. Hu, Z. Lin, and B. M. Chen, An analysis and design method for linear systems subject to actuator saturation and disturbance, Automatica 38 (2002), no. 2, 351-359.

[22] __ Analysis and design for discrete-time linear systems subject to actuator saturation, Systems \& Control Letters 45 (2002), no. 2, 97-112.

[23] H. Kimura, Pole assignment by gain output feedback, IEEE Transactions on Automatic Control 20 (1975), no. 4, 509-516.

[24] F. Mesquine and A. Benzaouia, Existence of output feedback for the regulator problem of a class of systems with constrained control, Proceedeings of the 1st International Conference on Electronics and Automatic, vol. 4, Tizi Ouzou, 1992, pp. 108-116.

[25] F. Mesquine, F. Tadeo, and A. Benzaouia, Regulator problem for linear systems with constraints on control and its increment or rate, Automatica 40 (2004), no. 8, 1387-1395.

[26] E. D. Sontag and H. J. Sussmann, Nonlinear output feedback design for linear systems with saturating controls, Proceedings of the 29th IEEE Conference on Decision and Control, vol. 6, Hawaii, 1990, pp. 3414-3416.

[27] V. L. Syrmos, C. Abdallah, and P. Dorato, Static output feedback: a survey, Proceedings of the 33rd IEEE Conference on Decision and Control, vol. 1, Florida, 1994, pp. 837-842.

A. Benzaouia: Department of Physics, EACPI Research Unit, Faculty of Sciences Semlalia, University of Cadi Ayyad, P.B. 2390, Marrakech, Morocco

E-mail address: benzaouia@ucam.ac.ma

F. Mesquine: Department of Physics, EACPI Research Unit, Faculty of Sciences Semlalia, University of Cadi Ayyad, P.B. 2390, Marrakech, Morocco

E-mail address: mesquine@ucam.ac.ma

A. Hmamed: Département de Physique and Laboratoire d'Electronique, Siganux, Systémes et d'Informatique, Faculté des Sciences Dhar El Mahraz, Université Sidi Mohamed Ben Abdellah, P.B. 1796, Fes, Morocco

E-mail address: hmamed_abdelaziz@yahoo.fr

H. Aoufoussi: Department of Electrical Engineering, École Supérieure de Technologie, Eljadida Km 7, Casablanca, Morocco

E-mail address: afoussi@iam.net.ma 


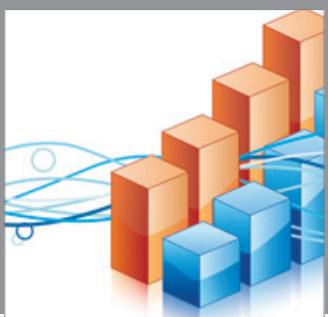

Advances in

Operations Research

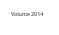

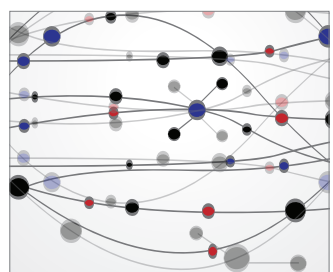

\section{The Scientific} World Journal
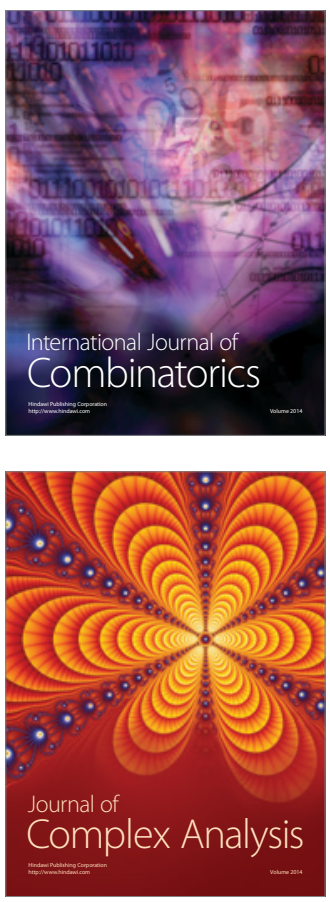

International Journal of

Mathematics and

Mathematical

Sciences
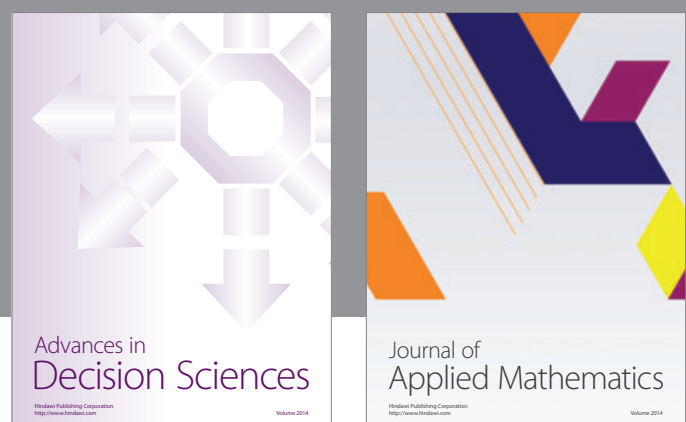

Journal of

Applied Mathematics
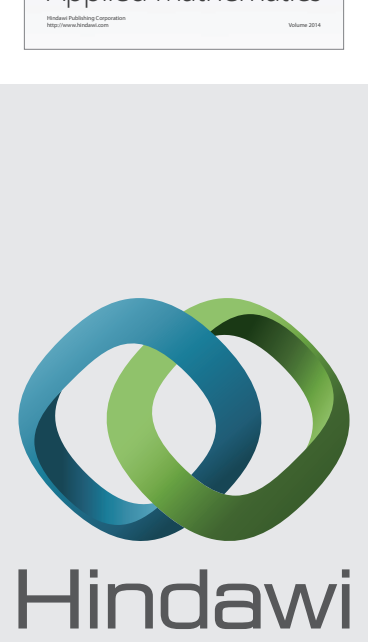

Submit your manuscripts at http://www.hindawi.com
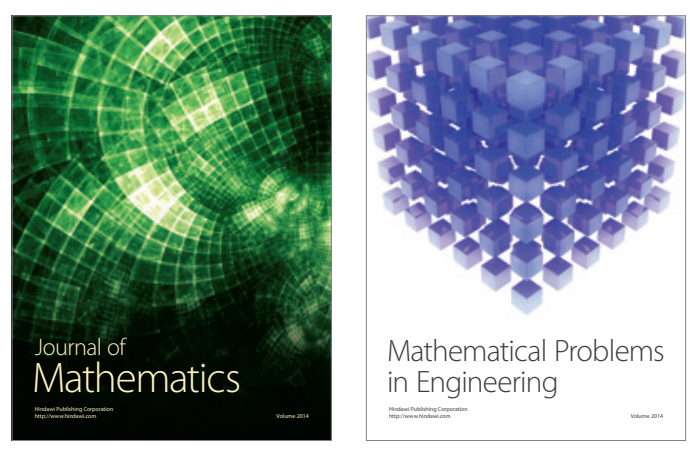

Mathematical Problems in Engineering
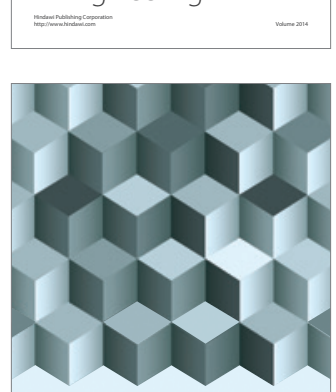

Journal of

Function Spaces
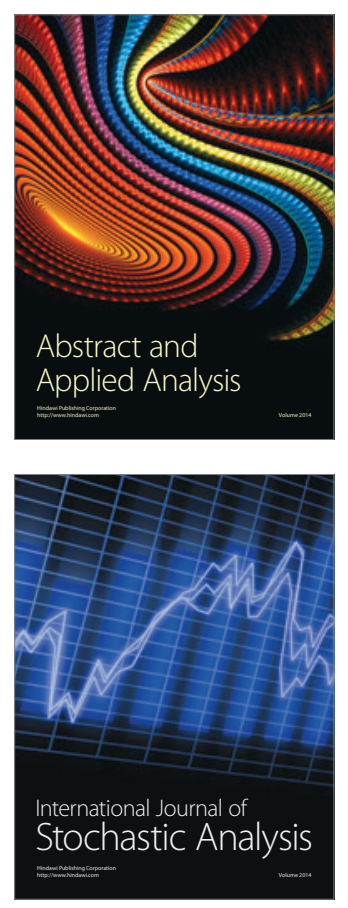

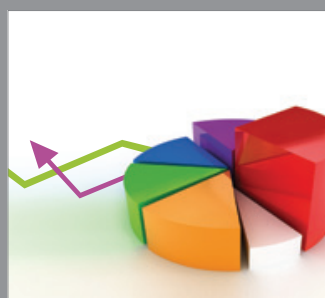

ournal of

Probability and Statistics

Promensencen
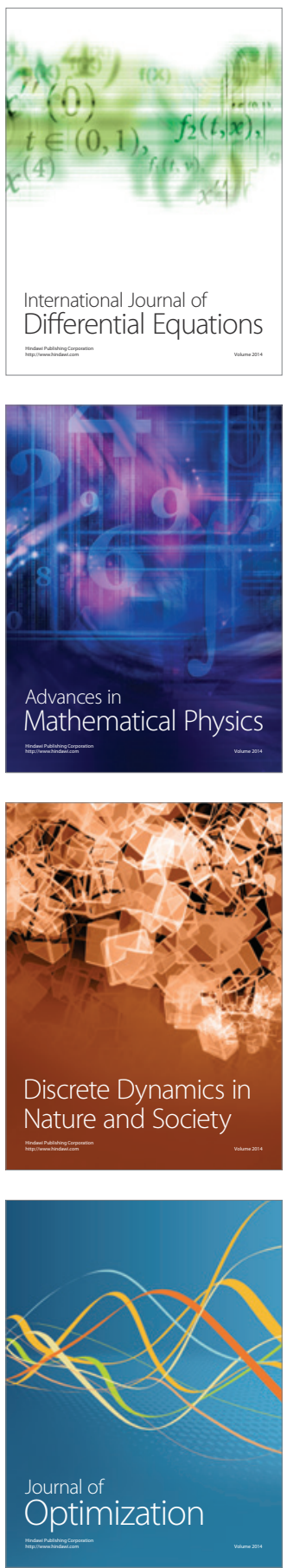